\title{
Incidence rates and case fatality rates of portal vein thrombosis and Budd-Chiari Syndrome
}

\author{
Walter Ageno ${ }^{1}$; Francesco Dentali'i' Fulvio Pomero2; Luigi Fenoglio ${ }^{2}$; Alessandro Squizzato'; Giovanni Pagani'; Roberta Re4; \\ Matteo Bonzini ${ }^{3}$ \\ ${ }^{1}$ Department of Clinical and Experimental Medicine, University of Insubria, Varese, Italy; ${ }^{2}$ Internal Medicine Department, S. Croce e Carle General Hospital, Cuneo, Italy; \\ ${ }^{3}$ Department of Clinical Science and Community Health, University of Milano, Italy; ${ }^{4}$ Department of Medicine, Ospedale Maggiore, Novara, Italy
}

\author{
Summary
}

Little information is available on the incidence of splanchnic vein thrombosis and on mortality rates during the acute phase of the disease. We performed a large epidemiologic study on hospital admissions for portal vein thrombosis (PVT) and the Budd-Chiari syndrome (BCS) between 2002 and 2012 in Northwestern Italy. Primary and secondary discharge diagnoses of PVT and BCS were identified using the 9th edition International Classification of Diseases codes 453.0, 572.1 and 452. Hospitalisations for recurrent events were not included. Information was collected on age and gender, vital status at discharge, duration of hospitalisation, and up to five secondary discharge diagnoses. Comorbidity was evaluated using the Charlson comorbidity index (CCI). A total of 3535 patients with PVT and 287 with BCS were hospitalized. The overall gender-specific incidence rates for PVT were 3.78 per 100,000 inhabitants in males and 1.73 per 100,000 inhabit- ants in females; for BCS 2.0 and 2.2 per million inhabitants, respectively. In-hospital case fatality was $7.3 \%$ in patients with PVT and $4.9 \%$ in patients with BCS. Age, non-abdominal solid cancer, and CCl were independently associated with in-hospital mortality in both PVT and BCS after stepwise regression analysis, male gender and haematologic cancer were associated with mortality in BCS patients only. In this large study we confirmed the low incidence of BCS and we found an incidence of PVT higher than previously reported. This incidence was stable during the period of observation. In-hospital mortality is not negligible, in particular in PVT patients.

\section{Keywords}

Portal vein thrombosis, Budd-Chiari syndrome, epidemiology, mortality

Received: October 15, 2016

Accepted after major revision: January 7, 2017

Epub ahead of print: February 9, 2017

https://doi.org/10.1160/TH16-10-0781

Thromb Haemost 2017; 117: 794-800

\section{Introduction}

The incidence of splanchnic vein thrombosis is poorly defined and estimates vary depending on data sources. Studies from Scandinavia have reported an incidence of portal vein thrombosis (PVT) ranging between less than 4 cases per million to 0.7 per 100.000 person-years, and an incidence of the Budd-Chiari syndrome (BCS) of 0.5 to 1 case per million people per year (1-3). Incidence rates appear to be higher in studies carried out in the 1990's than in studies conducted in the 1980's. Furthermore, the prevalence of PVT was reported to be of about $1 \%$ in a large study based on autopsies (4), and in a study based on consecutive abdominal computed tomography performed for reasons other than clinically suspected thrombosis (5). Thus, it is possible that the contemporary incidence of PVT is higher than previously reported and that PVT diagnoses, in particular with the increasing use of imaging tests in some patient populations, have been truly increasing over time.

A number of observational studies have reported on long-term mortality rates in patients with PVT and BCS (6-9), but little information is available on mortality rates during the acute phase of these diseases. In a recent population-based cohort study from Denmark, the 30-day risk of mortality in patients with splanchnic vein thrombosis was $20.6 \%$, and PVT had a stronger impact on mortality than BCS and mesenteric vein thrombosis (10). Whether this finding is related to the presence of multiple comorbidities in PVT patients requires additional investigation.

For these reasons, we conducted a large epidemiologic study to estimate the contemporary incidence of hospital admissions for PVT and BCS in Italy, to assess in-hospital mortality rates and factors associated with mortality, and also to provide data on changes in the incidence of these diseases over an 11-year time period, from 2002 to 2012.

\section{Materials and methods \\ Patient selection and eligibility}

The study was conducted in adherence to the Declaration of Helsinki, with the approval of the ethics committee of the coordinating center. Information on all hospital admissions for PVT and 
BCS between January 1, 2000 and December 31, 2012 in the Lombardy and Piedmont regions in Northwestern Italy (total population 13 millions) were obtained from the hospital discharge regional databases provided by each regional Center for Health Statistics.

Only patients with primary or secondary discharge diagnosis of PVT or BCS (on a total of five) were considered and identified using the following International Classification of Diseases, 9th Revision, Clinical Modification (ICD-9-CM) codes: 453.0 BCS, and 572.1 and 452 PVT.

For each hospital discharge record, the following variables were listed: gender, date of birth, date of hospital admission and discharge, department of admission and discharge, vital status at discharge, length of stay in the hospital (in days), and primary and up to five secondary discharge diagnoses. Patient names, addresses, or other potential identifiers were not reported in the database given by the Regional Centres for Health Statistics to comply with the national law dispositions in terms of privacy. An ad hoc developed identification number was used for each patient in order to allow the identification of repetitive hospital admissions.

To distinguish new cases (incident events) from recurrences, we only collected the first hospital admission with a diagnosis of PVT or BCS, during the entire study period. Because we did not have information before 2000, we excluded all events occurred during the first biennium of observation (2000-2001). Furthermore, hospitalised patients with a diagnosis of PVT or BCS and a previous hospital admission for the same diseases in 2000 and in 2001 for SVT were not considered as incident cases, and were excluded from the analysis. Thus, only patients with a first episode of PVT or BCS who were hospitalised between January 1, 2002 and December 31, 2012 were eligible for inclusion in this study. Finally, patients admitted to one hospital and then transferred to another hospital were counted as a single event with the date of hospitalisation referring to the admission hospital and the final diagnosis made by the discharging hospital.

\section{Data collection and analysis}

Data were expressed as absolute numbers, percentage, and mean with Standard Deviation (SD) and Interquartile Range (IQR, 25 and 75 percentile of the distribution). The annual incidence rates were calculated as the number of cases occurring in each year of the study period, divided by the number of total inhabitants in the two regions. Analyses were carried out considering PVT and BCS separately, calculating gender-specific incidence rates, and evaluating five years age categories by dividing incidence cases by the corresponding population according to the National Institute of Statistics. In the presence of concomitant diagnosis of PVT and BCS during the same hospital admission, we considered the disease reported in the primary discharge diagnosis (i. e. a total of 11 cases, 6 with PVT as the primary discharge diagnosis, 5 with BCS).

Age-standardised incidence rates were calculated based on the total Italian population.

Since we performed age-stratified analysis, we excluded patients younger than 20 years old at onset $(<0.3 \%$ of total cases) to obtain stable and not null age specific rates. Possible time trends in incidence rates across the study period (2002-2012) were evaluated for both genders using linear regression models, and confirmed using log transformation of age-standardised rates. Each annual rate was weighted according to the total number of incident cases.

Baseline characteristics and prevalence of concomitant diseases and of potential risk factors for PVT and for the BCS are presented separately for the two diseases. Risk factors were grouped in nine large categories according to the diagnostic discharge codes.

Comorbidity was evaluated using the Charlson comorbidity index (CCI) modified for ICD-9-CM database (11). This index allows the assessment of the impact of multimorbidity on in-hospital mortality, considering concomitant diagnoses of hypertension, obesity, diabetes mellitus, heart failure, peripheral arterial disease, history of cerebrovascular disease, chronic pulmonary disease, and malignancy. To obtain a sufficient numbers of subjects for the analysis, all patients with a CCI of equal to or greater than four were grouped together.

The prevalence of concomitant diseases and of potential risk factors in patients with PVT and BCS was compared by means of the Chi-square test, the Fisher exact test, or the Student t-test as appropriate.

In-hospital case fatality was calculated as the proportion of fatal cases (patients who died during hospitalisation) on the total of incident cases. Potential risk factors for mortality were evaluated in a multivariate model that included baseline characteristics (age and gender), thrombosis site (BCS vs PVT), and all the considered risk factors. Possible time trends in case fatality were assessed using logistic regression models separately for BCS and PVT.

Finally, a stepwise backward conditional binary logistic prognostic model was used to investigate independent risk factors for in-hospital mortality, separately for PVT and BCS. With this technique, all the variables were introduced in the model independently from the results of univariate analysis and were subsequently excluded step by step according to their level of significance (in details alpha1 $=0.10$ and alpha $2=0.20$ ). A second stepwise backward regression prognostic model was subsequently tested including the CCI as a potential independent variable and excluding risk factors for PVT or BCS from the model. Odds Ratios (ORs) and the corresponding 95\% Confidence Intervals (95\% CI) were presented for all the statistically significant variables. A two-tailed $\mathrm{p}<0.05$ was considered significant for all the analyses.

Statistical analyses were performed using STATA (Stata Corp, Austin, TX, USA).

\section{Results}

During the period of observation (2002-2012), a total of 3535 patients with PVT and 287 patients with BCS were hospitalised. Baseline characteristics of the two groups, risk factors and CCI are described in Table 1. Briefly, patients with PVT were older than patients with BCS (mean age 61 and 50 years, respectively, $\mathrm{p}<0.001$, median 64 and 50 years), and the prevalence of male 


\begin{tabular}{|c|c|c|c|c|}
\hline & All cases & BCS & PVT & P-value* \\
\hline Number of subjects & 3822 & 287 & 3535 & - \\
\hline Female gender & $35.1 \%$ & $54.4 \%$ & $33.6 \%$ & $<0.001$ \\
\hline $\begin{array}{l}\text { Age at hospital admission (years) } \\
\text { [mean (SD), Inter Quartile Range] }\end{array}$ & 60 (18), 51-73 & $50(21), 36-68$ & $61(17), 52-73$ & $<0.001$ \\
\hline $\begin{array}{l}\text { Duration of hospitalisation (days) } \\
\text { [mean (SD), Inter Quartile Range] }\end{array}$ & $15(19), 6-18$ & $17(21), 6-19$ & $14(18), 6-17$ & $<0.001$ \\
\hline \multicolumn{5}{|c|}{ Risk factors/co-morbidities reported in the same hospitalisation [n, \% on the total number of cases] } \\
\hline 1-Infectious diseases [n, (\%)] & $363(10 \%)$ & $49(17 \%)$ & $314(9 \%)$ & $<0.001$ \\
\hline 2-Autoimmune diseases [n, (\%)] & $20(0.5 \%)$ & $10(3 \%)$ & $10(0.3 \%)$ & $<0.001$ \\
\hline 3-Gastrointestinal cancer $[\mathrm{n},(\%)]$ & $1297(34 \%)$ & $19(7 \%)$ & $1278(36 \%)$ & $<0.001$ \\
\hline 4-Haematologic cancer [n, (\%)] & $40(1 \%)$ & $4(1 \%)$ & $36(1 \%)$ & 0.55 \\
\hline $5-0$ ther sites of cancer $[n,(\%)]$ & $197(5 \%)$ & $7(2 \%)$ & $190(5 \%)$ & 0.03 \\
\hline 6-Myeloproliferative neoplasms [n, (\% )] & $38(1 \%)$ & $9(3 \%)$ & $29(1 \%)$ & 0.002 \\
\hline $7-I B D[n,(\%)]$ & $15(0.4 \%)$ & $1(0.3 \%)$ & $14(0.4 \%)$ & 0.90 \\
\hline 8-Acute inflammatory diseases [n, (\%)] & $194(5 \%)$ & $9(3 \%)$ & $185(5 \%)$ & 0.12 \\
\hline 9-Cirrhosis [n, (\%)] & $1271(33 \%)$ & $39(14 \%)$ & $1232(35 \%)$ & 0.001 \\
\hline \multicolumn{5}{|l|}{ Charlson Comorbidity Index (CCI) } \\
\hline $\mathrm{CCl}=0$ & $1167(30 \%)$ & $176(61 \%)$ & $991(28 \%)$ & \\
\hline $\mathrm{CCl}=1$ & $546(14 \%)$ & $28(9 \%)$ & $518(15 \%)$ & \\
\hline $\mathrm{CCl}=2$ & $723(19 \%)$ & $20(7 \%)$ & $703(20 \%)$ & \\
\hline $\mathrm{CCl}=3$ & $722(19 \%)$ & $36(13 \%)$ & $686(19 \%)$ & \\
\hline $\mathrm{CCl}>=4$ & $664(17 \%)$ & $27(9 \%)$ & $637(18 \%)$ & $<0.001$ \\
\hline In hospital case-fatality rate & $7.1 \%$ & $4.9 \%$ & $7.3 \%$ & 0.13 \\
\hline
\end{tabular}

Table 1: Characteristics, risk factors and co-morbidities in the study population. Time period 2002-2012. gender was higher in the former group than in the latter $(66.4 \%$ and $45.6 \%$, respectively, $\mathrm{p}<0.001)$. A CCI equal to or greater than 4 was calculated in $18 \%$ and $9 \%$, respectively $(\mathrm{p}<0.001)$.

\section{Incidence rates for portal vein thrombosis}

The overall crude gender-specific incidence rates for the entire study period was 3.78 per 100,000 inhabitants in males and 1.73 per 100,000 inhabitants in females. This incidence raised with increasing age in both groups ( $\$$ Figure 1 ). The age-standardised incidence rate was 3.80 and 1.75 per 100,000 inhabitants, in males and females, respectively.

From 2002 to 2012, the crude incidence of PVT was substantially stable in both genders, with an incidence of 3.88 per 100,000 inhabitants in 2002 and 5.05 per 100,000 inhabitants in 2012 in males and an incidence of 1.59 per 100,000 inhabitants in 2002 and 2.24 per 100,000 inhabitants in 2012 in females ( Figure 2). Even with casual variation across years, a clear time trend was not evident ( $\mathrm{p}$-value 0.31 for males and 0.16 for females). Models with age-standardised incidence rates confirmed all results.

\section{Incidence rates for Budd-Chiari syndrome}

The overall crude incidence rate for the entire study period was 2.0 per million inhabitants in males and 2.2 per million inhabitants in females, respectively. This incidence did not change with increasing age in both groups. Trends in the incidence of BCS during the period of observation are reported, but no formal statistical analysis to assess variations was carried out due to the extremely low number of events.

\section{In-hospital case fatality and predictors of mortality}

The proportion of fatal cases was $7.3 \%$ in patients with PVT and $4.9 \%$ in patients with BCS ( $\mathrm{p}=0.13)$. In-hospital fatality rates according to the presence of major associated disorders are reported in Table 2. Bleeding was identified as the possible cause of death in 11 of 258 patients with PVT and in none of the 14 patients with BCS who died during hospitalisation. Time dependent trends for in-hospital mortality were assessed in both genders and across the entire study period. There were no significant trends observed for either PVT or BCS. 
Figure 1: Incidence of portal vein thrombosis according to age and gender $(A)$ and from 2002 to 2012 (B).

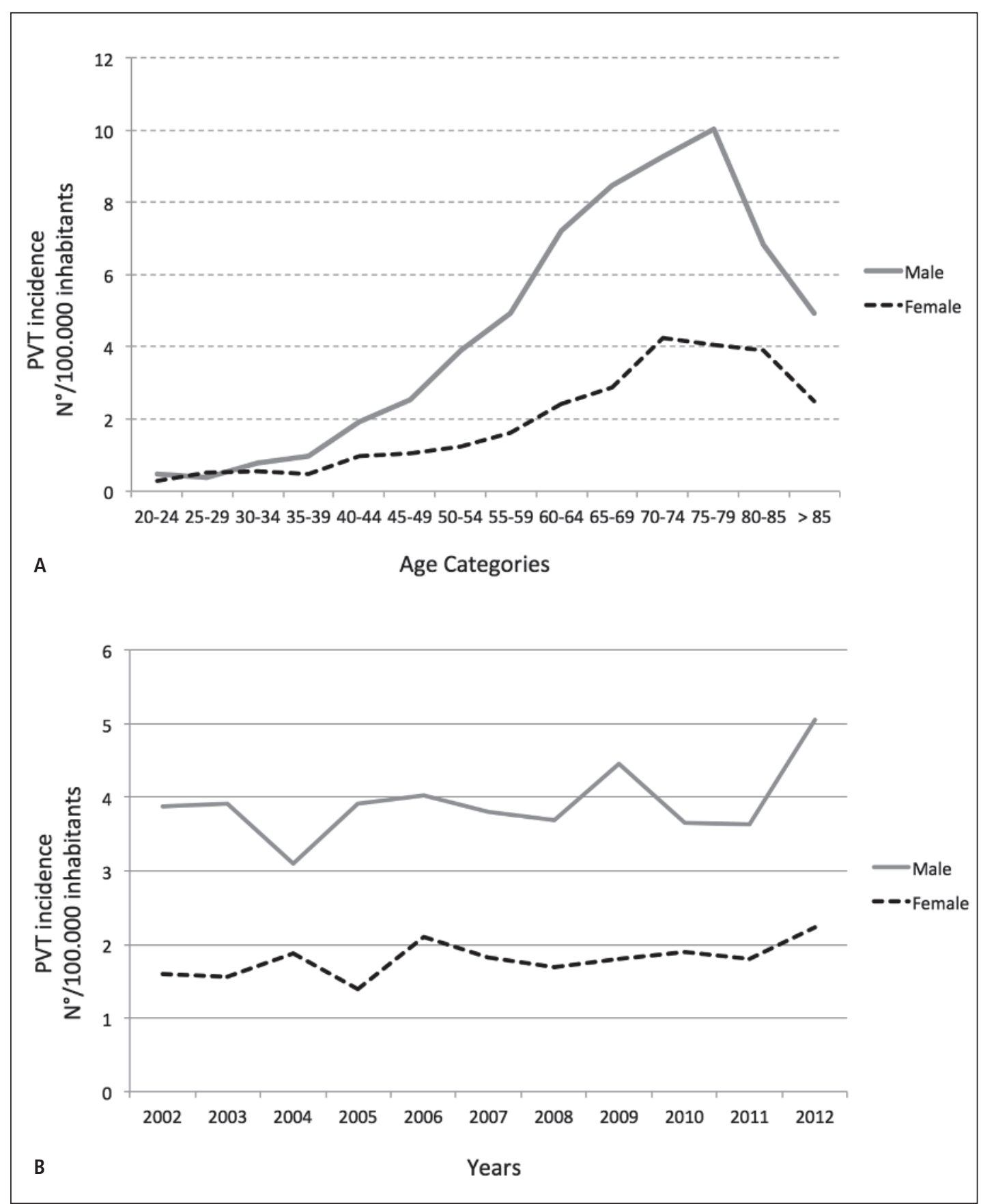

After stepwise backward regression analysis, the only independent risk factors for mortality in PVT patients using the first model including age, gender, site of thrombosis and all co-morbidities were age with an adjusted OR for one year increase of 1.04 (95\%CI: 1.03-1.05) and the presence of "other tumours" (i.e. non haematological or digestive tumours) during the same hospital record, with an adjusted OR of 2.55, 95\% CI: 1.71-3.80. Using the second model, which included age, gender, site of thrombosis and CCI, independent risk factors were age, adjusted OR 1.04 (95\% CI 1.03-1.05); and CCI, adjusted OR 1.43 (95\% CI 0.93-2.20) for CCI of 1, 1.61 (95\% CI 1.11-2.31) for CCI of 2, not significant (omitted by the model) for CCI of 3 , and 3.48 (95\% CI $2.50-4.80)$ for CCI of 4 or more.

For BCS, independent risk factors were age (OR 1.03, 95\% CI 0.99-1.06), male gender (OR 6.71, 95\% CI 1.65-27.3), concomitant presence of haematological cancer (OR 6.72, CI $95 \%$ 0.51-87.7), and the presence of "other tumours" (OR 6.72; 95\% CI 2.19-90.5). Using the second model, with CCI instead of single comorbidities, independent risk factors were age (OR 1.04, 95\% CI $1.00-1.07$ ), male gender (OR 4.17; 95\% CI 1.11-15.6) and CCI of 4 or more (OR 3.06, $95 \%$ CI 0.91-10.2). 


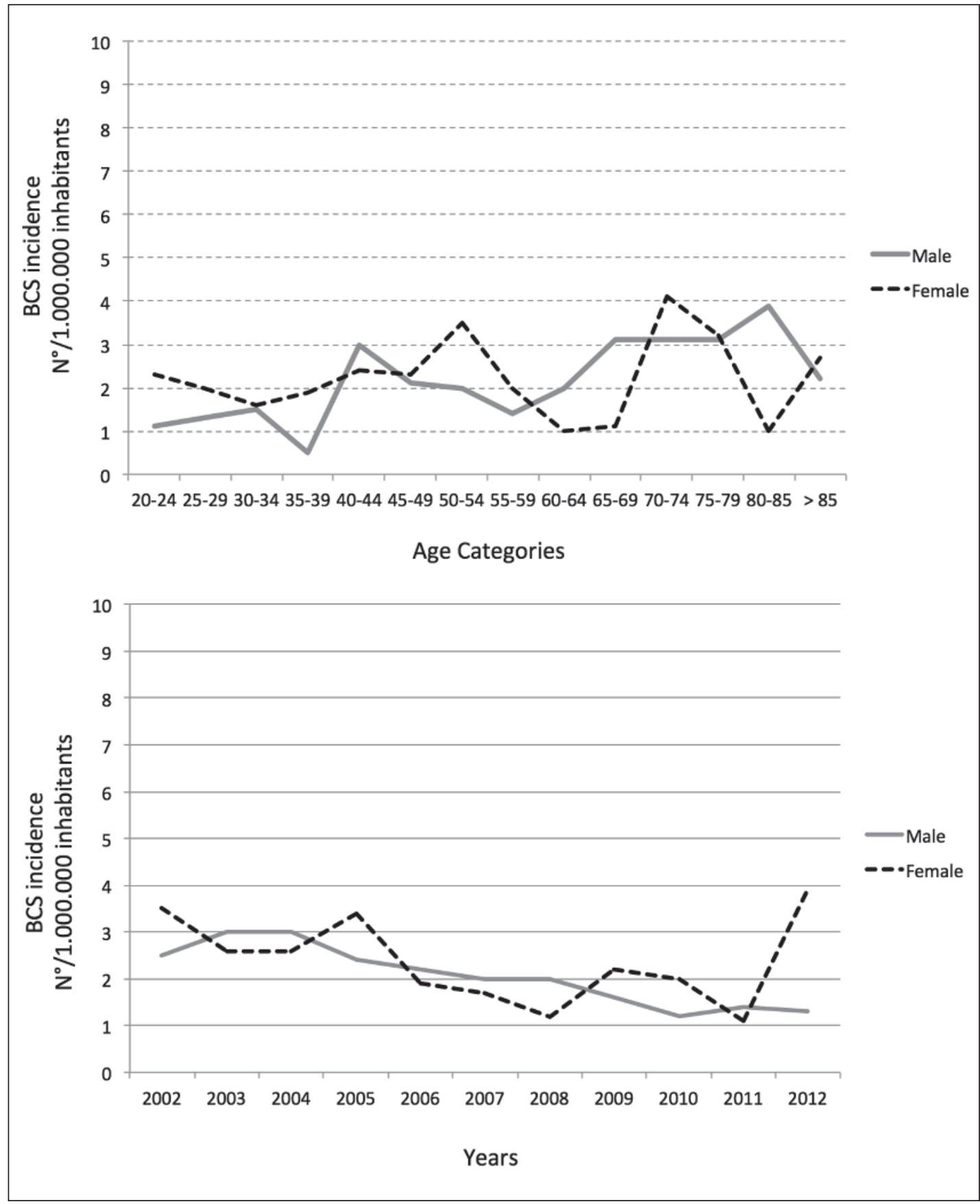

Figure 2: Incidence of Budd-Chiari syndrome according to age and gender $(A)$ and from 2002 to 2012 (B).

\section{Discussion}

To the best of our knowledge, this is the largest contemporary epidemiologic study carried out on PVT and BCS. Our results confirm that BCS is a rare disorder, with an incidence of about two cases per million inhabitants that is similar between genders and across age groups. Conversely, the incidence of PVT in this study is higher than previously reported $(1,2)$, is two times higher in males than in females, and increases with increasing age, with more than nine cases per 100,000 male inhabitants and more than four cases per 100,000 female inhabitants aged 70 to 80 years.
These incidence rates remained stable over the 11-year period of observation. Finally, the proportion of fatal cases was not negligible during a mean time of hospitalisation of approximately two weeks.

In the two previous epidemiologic studies from Sweden carried out between 1995 and 2003 for PVT and between 1990 and 2001 for BCS in a population of 4.4 million inhabitants, the incidence of PVT was 0.7 per 100,000 inhabitants and the incidence of BCS was 0.8 cases per million inhabitants $(2,3)$. In these studies, genderspecific incidences or in-hospital mortality rates were not reported. 
Table 2: In-hospital mortality in patients with risk factors/comorbidities.

\begin{tabular}{|c|c|c|c|c|c|c|}
\hline & \multicolumn{2}{|l|}{ All cases } & \multicolumn{2}{|l|}{ BCS } & \multicolumn{2}{|l|}{ PVT } \\
\hline & $\mathrm{n},(\%)$ & $\begin{array}{l}\text { Case } \\
\text { fatality }\end{array}$ & $n,(\%)$ & $\begin{array}{l}\text { Case } \\
\text { fatality }\end{array}$ & $\mathrm{n},(\%)$ & $\begin{array}{l}\text { Case } \\
\text { fatality }\end{array}$ \\
\hline \multicolumn{7}{|c|}{ Risk factors/co-morbidities reported in the same hospitalisation } \\
\hline 1-Infective diseases & $363(10 \%)$ & $3 \%$ & $49(17 \%)$ & - & $314(9 \%)$ & $3 \%$ \\
\hline 2-Autoimmune diseases & $20(0.5 \%)$ & $10 \%$ & $10(3 \%)$ & - & $10(0.3 \%)$ & $20 \%$ \\
\hline 3-Gastrointestinal cancer & $1297(34 \%)$ & $9 \%$ & $19(7 \%)$ & $10 \%$ & $1278(36 \%)$ & $9 \%$ \\
\hline 4-Haematologic cancer & $40(1 \%)$ & $8 \%$ & $4(1 \%)$ & - & $36(1 \%)$ & $6 \%$ \\
\hline 5-0ther sites of cancer & $197(5 \%)$ & $19 \%$ & $7(2 \%)$ & $43 \%$ & $190(5 \%)$ & $18 \%$ \\
\hline 6-Myeloproliferative neoplasms & $38(1 \%)$ & $5 \%$ & $9(3 \%)$ & - & $29(1 \%)$ & $7 \%$ \\
\hline 7-IBD & $15(0.4 \%)$ & $0 \%$ & $1(0.3 \%)$ & - & $14(0.4 \%)$ & - \\
\hline 8-Acute Intestinal diseases & $194(5 \%)$ & $7 \%$ & $9(3 \%)$ & - & $185(5 \%)$ & $7 \%$ \\
\hline 9-Cirrhosis & $1271(33 \%)$ & $7 \%$ & $39(14 \%)$ & - & $1232(35 \%)$ & $7 \%$ \\
\hline
\end{tabular}

Previous observational cohort studies did not report substantial differences in gender prevalence in PVT patients (7), but reported a higher prevalence of female gender in the BCS population (between $57 \%$ and $65 \%)(7,9)$. Furthermore, patients enrolled in observational studies were younger than in the present epidemiologic study. One study reported a mean age of PVT patients of 54 years (7), and one study reported a median age of PVT patients of 43 years (12), while for BCS patients one study reported a mean age of 40 years (7) and another study reported a median age of 38 years (9). These differences could be explained by a referral bias in these observational cohorts or a selection bias due to the exclusion, for example, of patients who died before hospital discharge or with a short life expectancy. Another explanation is that our study failed to capture younger patients with no associated comorbidities who may have been entirely managed out of hospital.

The relative incidence of risk factors for PVT and BCS varies with age, gender, economic status, geographical area, and, not least, the site of thrombosis (5). While local predisposing factors such as liver cirrhosis, abdominal cancer, or intraabdominal inflammatory conditions are common in patients with PVT, systemic risk factors, including hematologic disorders, autoimmune diseases, and the use of hormonal therapy, are the most common risk factors for BCS (5). In our study, based on the collection of ICD-9 diagnoses, the prevalence of some common factors associated with splanchnic vein thrombosis, such as liver cirrhosis or solid abdominal cancer, was consistent with the prevalence reported in previous observational studies, but this was not the case for other risk factors such as myeloproliferative neoplasms or other haematologic malignancies. Whether this is due to underreporting of some associated disorders or to a lower rate of hospitalisations in these patient populations is unclear. In addition, other risk factors such as hormonal therapy could clearly not be collected in this type of study. However, the aim of this study was not to gather information on the prevalence of risk factors for PVT or BCS, since this information is sufficiently consistent in the literature, but to assess for the first time the role of concomitant disorders on the risk of in-hospital mortality. Previous studies have reported age, cancer, myeloproliferative neoplasms as independent predictors of long-term mortality $(2,7,8)$, in this study we found that age, nonabdominal solid cancer, and multiple co-morbidities assessed by means of CCI were independently associated with short-term mortality in both PVT and BCS, while male gender and haematological cancer were associated with mortality in BCS patients only.

We have previously described the epidemiology of pulmonary embolism (PE) in the same regions of Northern Italy (13). Patients hospitalised for PE (mean age 72.8 years) were more than 10 years older than patients with PVT and more than 20 years older than patients with BCS. The mean duration of hospitalisation was similar (14 days for PE patients), but the burden of comorbidities was lower, given that a CCI of 4 or more was found in a lower proportion of patients $(4.8 \%)$. The crude incidence rate for PE was ap-

\section{What is known about this topic?}

- Little information is available on the incidence of portal vein thrombosis and Budd-Chiari syndrome.

- The results of autoptic studies and in studies conducted on consecutive abdominal CT scans performed for reasons other than the suspicion of splanchnic vein thrombosis suggest that this disease may be more frequent than previosly reported.

- Temporal trends in the incidence of portal vein thrombosis and Budd Chiari syndrome are unknown.

\section{What does this paper add?}

- This large study provides a contemporary estimate of the incidence of portal vein thrombosis and Budd Chiari syndrome in a Western country and provides information on inhospital case fatality rates of these diseases. 
proximately 41 cases and 55 cases per 100,000 inhabitants for males and females, respectively, which is more than 10 times the incidence of PVT. Differently from what we observed for PVT and BCS patients, the incidence of PE significantly increased over time during the period of observation. Finally, the in-hospital case fatality rate for PE was higher than that for PVT or BCS (13.3\%).

There are strengths and limitations in this study that need to be acknowledged. Strengths include the very large sample of the analysed population that enabled us to collect information on a very large group of patients with PVT and BCS, and the long period of observation. The main limitation is intrinsic to the use of ICD9-CM diagnosis codes, which may have a low sensitivity and specificity in the identification of patients hospitalised for these diseases. To avoid false positive diagnoses, we only included patients with first and second codes of the diseases. Further, because of the design of our study, a number of events that did not result in hospitalisation have been obviously missed. The proportion of patients with PVT or BCS who are entirely treated out of hospital is currently unknown, but patients with a diagnosis of chronic disease, or patients with incidentally detected thrombosis, in particular when the portal vein is affected, may be managed conservatively $(5,14)$. This is particularly important for incidentally diagnosed events, given their non-negligible rates of detection and recommendations to not routinely use anticoagulant treatment $(5,6)$. Unfortunately, available information using disease codes in the present study does not allow to discriminate between symptomatic and asymptomatic events, and the management of incidentally detected SVT is likely heterogeneous. In a recent study, we found that the majority of patients with asymptomatic SVT actually received anticoagulant treatment, and that outcome rates were similar to those observed in symptomatic patients $(14,15)$. For this reason, we can expect that at least some, but likely not all patients with incidentally detected SVT required in-hospital management. If our hypothesis holds true, the results this study may likely underestimate the incidence of these diseases, further supporting our finding of a higher incidence of PVT than previously reported. Finally, due to the design of the study and the unavailability of information on therapeutic strategies in our source databases, we could not assess the impact of antithrombotic drugs on mortality rates.

In conclusion, the results of this large epidemiologic study confirm the low incidence of BCS and report an incidence of PVT that is higher than previously reported, but stable during the eleven years of observation. We also report for the first time proportion of fatal cases and factors associated with in-hospital mortality in patients with PVT and BCS. These results improve our knowledge of these unusual disorders and may help researchers in designing future studies in the field.

\section{Conflicts of interest}

None declared.

\section{References}

1. Almdal TP, Sorensen TI. Incidence of parenchymal liver diseases in Denmark, 1981 to 1985: analysis of hospitalisation registry data. The Danish Association for the Study of the Liver. Hepatology 1991; 13: 650-655.

2. Rajani R, Bjornsson E, Bergquist A, et al. The epidemiology and clinical features of portal vein thrombosis: a multicentre study. Aliment Pharmacol Ther 2010; 32: 1154-1162.

3. Rajani R, Melin T, Bjornsson E, et al. Budd-Chiari syndrome in Sweden: epidemiology, clinical characteristics and survival-an 18-year experience. Liver Int 2009; 29: 253-259.

4. Ögren M, Bergqvist D, Bjo囚rck M, et al. Portal vein thrombosis: Prevalence, patient characteristics and lifetime risk: A population study based on 23796 consecutive autopsies. World J Gastroenterol 2006; 12: 2115-2119.

5. Ageno W, Dentali F, Squizzato A. How I treat splanchnic vein thrombosis. Blood 2014; 124: 3685-3691.

6. Ageno W, Squizzato A, Togna A, et al. Incidental diagnosis of deep vein thrombosis in consecutive patients undergoing a CT scan of the abdomen: a retrospective cohort study. J Thromb Haemost 2012; 10: 158-160.

7. Thatipelli MR, McBane RD, Hodge DO, Wysokinski WE. Survival and recurrence in patients with splanchnic vein thromboses. Clin Gastroenterol Hepatol 2010; 8: 200-205.

8. Ageno W, Riva N, Schulman S, et al. Long-term Clinical Outcomes of Splanchnic Vein Thrombosis: Results of an International Registry. JAMA Intern Med 2015; 175: 1474-1480.

9. Darwish Murad S, Plessier A, Hernandez-Guerra M, et al. (European Network for Vascular Disorders of the Liver). Etiology, management, and outcome of the Budd-Chiari syndrome. Ann Intern Med 2009; 151: 167-175.

10. Sogaard KK, Darvalics B, Horvath-Puho E, Sorensen HT. Survival after splanchnic vein thrombosis: a 20 -year nationwide cohort study. Thromb Res 2016; Epub ahead of print.

11. Sundarayayan V, Henderson T, Perry C, et al. New ICD-10 version of the Charlson comorbidity index predicted in-hospital mortality. J Clin Epidemiol 2004; 57: 1288-1294.

12. Condat B, Pessione F, Hillaire S, et al. Current outcome of portal vein thrombosis in adults: risk and benefit of anticoagulant therapy. Gastroenterology 2001; 120: 490-497.

13. Dentali F, Ageno W, Pomero F, et al. Time trends and case fatality rate of pulmonary embolism during 11 years of observation in Northwestern Italy. Thromb Haemost 2016; 115: 399-405.

14. Ageno W, Riva N, Schulman S, et al. Antithrombotic treatment of splanchnic vein thrombosis: results of an International registry. Semin Thromb Hemost 2014; 40: 99-105.

15. Riva N, Ageno W, Schulman S, et al. Clinical history and antithrombotic treatment of incindentally detected splanchnic vein thrombosis: a multicentre international prospective registry. Lancet Haematol 2016; e267-275. 